\title{
Estado ecológico del río Oja (cuenca del Ebro, La Rioja, España), mediante indicadores de macroinvertebrados
}

\author{
María Valladolid $^{1, *}$, Mercedes Arauzo $^{2}$ y Juan José Martínez-Bastida ${ }^{2}$ \\ ${ }^{1}$ Dpto. Biodiversidad y Biología Evolutiva, Museo Nacional de Ciencias Naturales (CSIC), c/José Gutiérrez \\ Abascal, 2. 28006 Madrid. Fax: 34915645078. \\ 2 Dpto. Contaminación Ambiental. Instituto de Ciencias Agrarias. Centro de Ciencias Medioambientales \\ (CSIC), c/ Serrano 115 dpdo., 28006 Madrid, Spain. Fax: 349156408 00. e-mails: mercedes@ccma.csic.es, \\ Martinez.Bastida@ccma.csic.es
}

* Autor responsable de la correspondencia: marval@mncn.csic.es

Recibido: $23 / 2 / 10$

Aceptado: $21 / 7 / 10$

\begin{abstract}
Ecological status of the Oja River (Ebro Basin, La Rioja, Spain) based on macroinvertebrate indexes

This work provides results about the water quality of the Oja or Glera River (Oja-Tirón basin) during the years 2003-2007, as well as information from the historical data taken from previous basin reports (2004 report, that includes data from 1994 to 2004 and 2007 report). Seven sampling points have been selected, distributed between the three typologies defined in the Oja River: in ecotype 11, Siliceous Mediterranean mountain river, 3 points; in ecotype 26, Humid calcareous mountain river, 1 point and in ecotype 12, Mediterranean calcareous mountain rivers, 3 points. Several macro invertebrate indexes (IBMWP, IASPT, NFAM, NFPLE and EPT) and their ecological quality ratios (EQR) were calculated for each point.

As a whole, $75.86 \%$ of the IBMWP measurements show Very good or Good quality, with a $13.79 \%$ of Moderate quality, meanwhile $10.35 \%$ of them appear as Deficient.

Regarding its historic evolution, in those stretches of the river which present permanent water a progressive improvement of water quality was observed from the first available data (years 1994-95) until the most recent ones (2007), according to the most recent values of water quality classification for IBMWP (BOE, 2008).
\end{abstract}

Key words: Oja River, ecological status, macroinvertebrate, IBMWP, Water Framework Directive.

\section{RESUMEN}

Estado ecológico del río Oja (cuenca del Ebro, La Rioja, España), mediante indicadores de macroinvertebrados

El presente trabajo presenta resultados sobre el estado ecológico del río Oja o Glera (cuenca del Oja-Tirón) durante los años 2003-2007, así como información proveniente de datos históricos recopilados a partir de los informes de cuenca existentes (informe de 2004, que incluye datos desde 1994 hasta 2004 e informe de 2007). Se han seleccionado 7 puntos, distribuidos entre las tres tipologías definidas en el río Oja: en el ecotipo 11, ríos de montaña mediterránea silícea, 3 puntos; en el ecotipo 26, ríos de montaña húmeda calcárea, 1 punto y en el ecotipo 12, Ríos de montaña mediterránea calcárea, 3 puntos. Para cada punto se han calculado una serie de índices de macroinvertebrados (IBMWP, IASPT, NFAM, NFPLE y EPT) y sus ratios de estado ecológico (EQR).

En conjunto, el $75.86 \%$ de las medidas del IBMWP dan una calidad Muy buena o Buena y un $13.79 \%$ Moderada mientras que el $10.35 \%$ aparece como Deficiente.

En cuanto a su evolución histórica, en aquellos tramos del río que presentan agua de manera permanente se observó una mejora progresiva de la calidad del agua desde los primeros datos de los que se dispone (años 1994-95) hasta los más recientes (2007), según los valores más recientes de clasificación de calidad de agua para el IBMWP (BOE, 2008).

Palabras clave: Río Oja, estado ecológico, macroinvertebrados, IBMWP, Directiva Marco del Agua. 


\section{INTRODUCCIÓN}

Los objetivos prioritarios de la Directiva Marco del Agua (DMA, Directiva 2000/60/CE) para alcanzar un buen estado de calidad de agua son: prevenir el deterioro, proteger, mejorar, regenerar y reducir la contaminación de las masas de agua, eliminando o suprimiendo gradualmente los vertidos (BOE, 2008). Para ello, los Estados miembros de la Unión Europea deberán identificar y caracterizar las cuencas fluviales, incluyendo la incidencia de las actividades humanas (presiones e impactos) y un registro de las zonas a proteger. Se completará con la monitorización de las masas de agua y la implementación de medidas para alcanzar los objetivos de calidad de la DMA, en el caso de aquellas masas de agua que no los cumplan. Esta información aparecerá resumida en un plan hidrológico de cuenca, que podrá complementarse con programas y planes hidrológicos más detallados para cada subcuenca.

La Confederación Hidrográfica del Ebro (CHE), junto con el Centro de Estudios y Experimentación de Obras Públicas (CEDEX), inició en los años 1990-93 estudios basados en indicadores biológicos (índices de macroinvertebrados), que sirvieron para la puesta en marcha de la Red de Control de Variables Ambientales (RCVA). En su página Web se pueden encontrar los informes relacionados con la evolución del cumplimiento de los objetivos de la DMA, como p.e. los protocolos de muestreo y análisis para los invertebrados bentónicos (CHE, 2005 a), el establecimiento de las condiciones de referencia (CHE, 2006 a), el borrador del Plan Hidrológico de la cuenca del Oja-Tirón (Confederación Hidrográfica del Ebro, Junta de Castilla y León y Gobierno de La Rioja, 2007) o los informes de calidad de las aguas (CHE 2004, 2005 b, 2006 b, 2007, 2008). Por su parte, el Gobierno de La Rioja (GLR) en su página Web recoge los documentos referidos a la incidencia de las actividades humanas como p.e. los Planes Directores de Saneamiento y Depuración de La Rioja (BOR, 2001; GLR, 2008) o el Documento Técnico de la cuenca del Oja-Tirón (GLR, 2007).

Por otra parte, se han publicado resultados de la calidad de agua en la demarcación del Ebro basados en estos informes, como p.e. los de Oscoz et al. $(2007,2008)$ que aportan una visión general del estado de los ríos de la cuenca del Ebro en conjunto, así como una serie de artículos sobre diversos aspectos del río Oja, como su fauna de macroinvertebrados acuáticos (Martínez-Bastida et al., 2006; Valladolid et al., 2006, 2007, 2010) o la calidad de las aguas del acuífero aluvial (Arauzo et al., 2006; Martínez-Bastida et al., 2007).

Con este trabajo queremos ampliar la información sobre la calidad del agua del río Oja (índices de macroinvertebrados), integrando tanto datos propios (2003-2007) como históricos (informe de la CHE de 2004, con datos desde 1994 hasta 2004, e informe de la CHE de 2007), completando la información de aquellos años donde nose dispone de datos de este río. Por último, se presenta la evolución temporal que ha experimentado la calidad del agua del río Oja (IBMWP, Alba-Tercedor et al., 2002) durante el periodo 1994-2007.

\section{ÁREA DE ESTUDIO}

El río Oja o Glera nace en la Sierra de la Demanda (Sistema Ibérico) y desemboca en el río Tirón, afluente del Ebro por la derecha (Fig. 1). La cuenca del Oja-Tirón se extiende por las comunidades de La Rioja (Rioja Alta) y Castilla-León (Burgos). De régimen pluvionival con influencia oceánica, presenta dos zonas diferenciadas: la cabecera, con clima montañoso húmedo y el tramo medio-bajo, con clima mediterráneo continental. En cabecera dispone de una cubierta nival y derrubios periglaciales que actúan como reservas hídricas en verano, aunque no evitan un fuerte estiaje en agosto. A partir de Ezcaray el río se abre en un amplio valle aluvial con una alta permeabilidad hacia el acuífero, que unido a la extracción intensiva de agua hace que el río desaparezca totalmente en la parte media del valle durante las épocas de menor caudal (finales de primavera-verano hasta principios de otoño, dependiendo del año). En Castañares surge de nuevo el río por la salida natural del acuífero, que le permite mantener un caudal regular hasta su desembocadura en el río Tirón.

En el río Oja se encuentran tres tramos fluviales diferentes (MIMAN, 2005; BOE 2008): eco- 


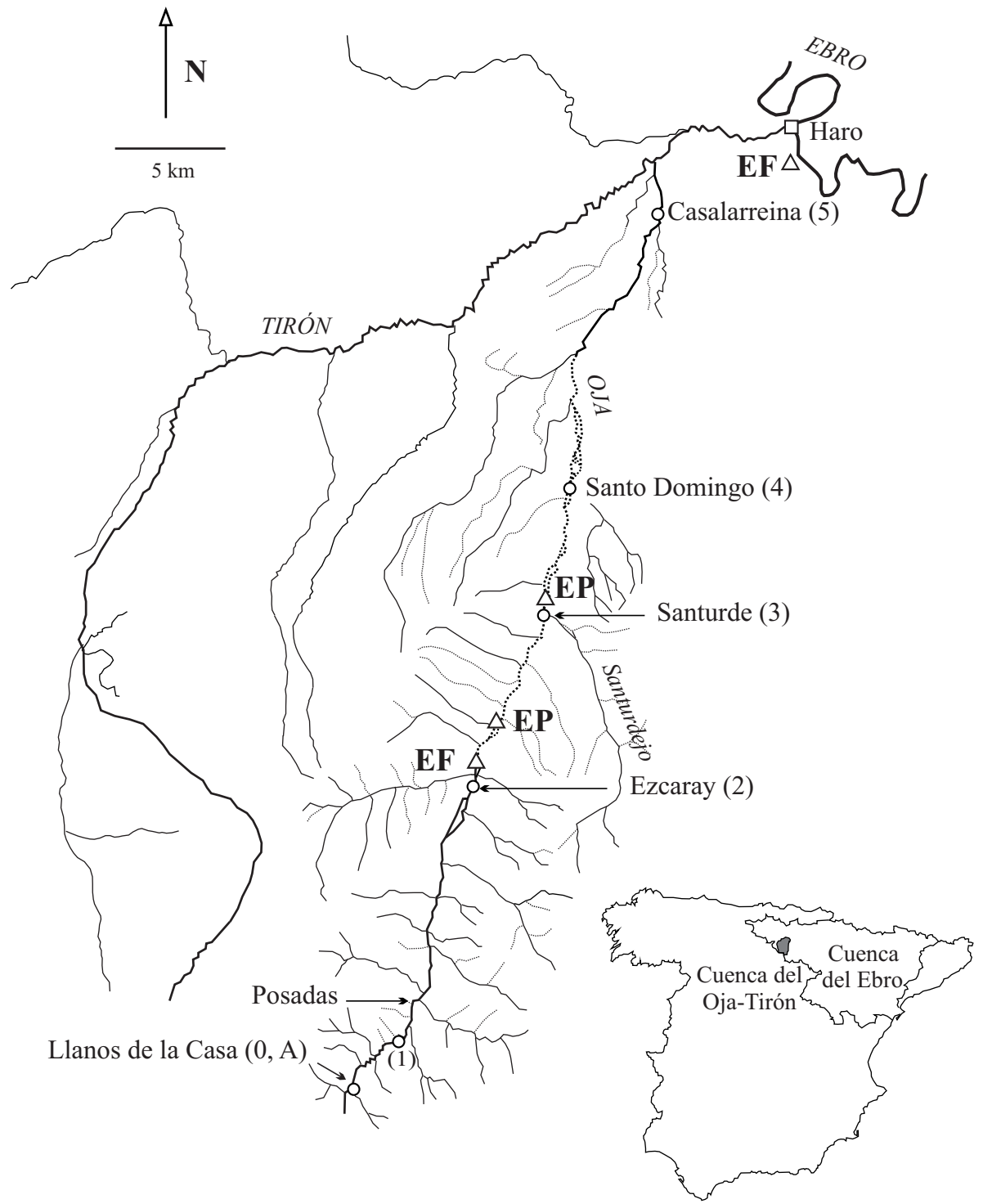

Figura 1. Localización de la cuenca del río Oja-Tirón y de los puntos de muestreo (ver Tabla 1). EF = EDARs en funcionamiento (Ezcaray, Haro), EP = EDARs en proyecto o en ejecución (Ojacastro, Santurde). Location of the Oja-Tirón River basin and the sampling points (see Table 1). EF= EDARs working (Ezcaray, Haro), EP=EDARs planned or being built (Ojacastro, Santurde).

tipo 11, Ríos de montaña mediterránea silícea; ecotipo 26, Ríos de montaña húmeda calcárea y ecotipo 12, Ríos de montaña mediterránea calcárea. El tramo alto de la cuenca (ecotipos 11 y 26) está recubierto por bosques mixtos y se dedica principalmente a la ganadería, mientras que los tramos medios-bajos discurren por un amplio valle aluvial, dedicado a la agricultura tanto de secano como de regadío (hortofrutícola).
En el Plan Hidrológico de la cuenca del Oja-Tirón (Confederación Hidrográfica del Ebro, Junta de Castilla y León y Gobierno de La Rioja, 2007) las principales presiones identificadas son: la extracción de agua para abastecimiento (tramo de cabecera básicamente) y regadío (principalmente en áreas del acuífero aluvial, en tramos medio y bajo), la contaminación de tipo difuso (cultivos, ganadería) o puntual (vertidos urbanos 
Tabla 1. Localización de los puntos de muestreo. Los datos de los puntos 0 y A se han calculado a partir de la Hoja ${ }^{\circ} 21-11$ (240), Ezcaray (Cartografía militar de España, E: 1: 50.000, Serie L. 1989). P: punto de muestreo. Tipo de río: tipología (BOE, 2008). Location of the sampling points. Data of points 0 and A have been calculated from the Map No. 21-11 (240), Ezcaray (Spanish Military Cartography, Scale 1: 50.000, L Series, 1989). P: sampling point. Tipo de río: typology (BOE, 2008).

\begin{tabular}{llccl}
\hline P & \multicolumn{1}{c}{ Localización } & Coordenadas UTM & Altitud (m) & \multicolumn{1}{c}{ Tipo de río } \\
\hline A & Llanos de la Casa, afluente & 30T 493900 UTM 4673000 & 1200 & 11. Montaña mediterránea silícea \\
0 & Llanos de la Casa & 30T 493900 UTM 4673000 & 1200 & 11. Montaña mediterránea silícea \\
1 & Posadas & 30T 494300 UTM 4687373 & 1000 & 11. Montaña mediterránea silícea \\
2 & Ezcaray & 30T 499341 UTM 4687339 & 790 & 26. Montaña humeda calcárea \\
3 & Santurde & 30T 503289 UTM 4699294 & 711 & 12. Montaña mediterránea calcárea \\
4 & Sto. Domingo de la Calzada & 30T 503323 UTM 4699202 & 616 & 12. Montaña mediterránea calcárea \\
5 & Casalarreina & 30T 507069 UTM 4710818 & 480 & 12. Montaña mediterránea calcárea \\
\hline
\end{tabular}

eindustriales) y las modificaciones en la morfología del cauce por la extracción de áridos en tramos medios (p.e. en Santo Domingo de la Calzada).

Por otra parte, en los años 2002-03 se pusieron en marcha distintos sistemas de depuración, como fosas sépticas y decantadores en pueblos de cabecera de menos de 100 habitantes, y los EDAR de Ezcaray y Haro. El primero recoge las aguas desde cabecera hasta Ezcaray, mientras que el de Haro conecta mediante un colector gran parte de las poblaciones del valle por debajo de Ezcaray. En estos momentos está prevista la construcción de dos EDAR adicionales por debajo de Ezcaray, uno en Ojacastro (en proyecto) y otro en Santurde (en ejecución), que recogerá las aguas del río Santurdejo.

Un problema importante del acuífero del Oja, es la presencia de valores altos de nitratos en su tramo inferior, que provienen de la agricultura existente en la zona medio-baja del valle. La alta permeabilidad de los terrenos aluviales, junto con los rápidos desplazamientos del agua en el interior del acuífero hace que los contaminantes se acumulen en el extremo norte del mismo, que actúa como un fondo de saco (Arauzo et al., 2006). En Castañares parte de este agua surge hacia el Oja, especialmente en los meses de sequía cuando se interrumpe el flujo aguas arriba y prácticamente todo el agua circulante proviene del acuífero.

\section{MATERIAL Y MÉTODOS}

En 2003 se seleccionaron 5 puntos a lo largo del cauce del río (1 a 5) y dos más en cabecera en
2005, uno en el río Oja (0) y otro en un afluente (A) (Tabla 1). Con el fin de analizar la evolución de la calidad del agua se utilizaron también datos procedentes de los infomes realizados por la CHE en 2004 (que incluye los valores de IBMWP desde 1994 hasta 2004) y en 2007. En ellos aparecen 4 puntos de control en este río: 1. Azarrulla (por debajo del punto 1), 2. Ezcaray, 3. Santo Domingo de la Calzada y 4. Casalarreina, estos tres últimos coincidentes con los de nuestro estudio. Los puntos A, 0, 1 y Azarrulla pertenecen al ecotipo 11 (Ríos de montaña mediterránea silícea), el punto 2 al ecotipo 26 (Ríos de montaña húmeda calcárea) y los puntos 3,4 y 5 al ecotipo 12 (Ríos de montaña mediterránea calcárea) (MIMAN, 2005; BOE, 2008).

Para la recogida de los macroinvertebrados se utilizó un dispositivo tipo surber $30 \times 30 \mathrm{~cm}$, con una manga de nytal de $250 \mu \mathrm{m}$ de luz de ma1la. Las muestras de 2003-2004 se recogieron de manera cuantitativa, en un transecto con varias réplicas (de 3 a 12, dependiendo del punto y la estación), el resto de las muestras se recogió de manera semicuantitativa, con periodos de muestreo de 30 minutos y seleccionando los distintos tipos de hábitats presentes en cada punto. Las muestras se fijaron en el campo con formol al $4 \%$ tamponado con ClNa al $0.9 \%$. En el laboratorio las muestras se separaron y los macroinvertebrados se conservaron en alcohol al $70 \%$, determinándose a nivel de familia mediante claves generales (Tachet et al., 2003).

Basándonos en el informe de la CHE (2006 a), donde se valida la idoneidad de los distintos índices de macroinvertebrados utilizados hasta esa 
fecha, se calcularon los índices NFAM (Número total de Familias), EPT (Número de Familias de Efemerópteros, Plecópteros y Tricópteros), NFPLE (Número de Familias de Plecópteros), IASPT (Iberian Average Score Per Taxon, Armitage et al., 1983) e IBMWP (Iberian Biological Monitoring Working Party, Alba-Tercedor et al., 2002), así como la ratio de estado ecológico
(EQR) en cada tramo fluvial. Los límites de clase se han calculado a partir de los valores de referencia para cada ecotipo (Tabla 2) incluidos en el informe de la CHE (2006 a). Para el IBMWP se han utilizando para el cálculo los valores de referencia más actualizados (BOE, 2008).

Por último, para estudiar la evolución de la calidad del agua, junto con los datos propios se

Tabla 2. Condiciones de referencia y límites de clase de estado ecológico para los distintos índices según el tipo de río. CR: condiciones de referencia.V: valores límite, EQR: ratios de estado ecológico. Puntos: puntos de muestreo incluidos en el tipo de río (ver Tabla 1). Los valores del IBMWP se han calculado a partir de las condiciones de referencia del BOE 2008, los valores reseñados como IBMWP(1) y las condiciones de referencia del resto de los índices son los utilizados en CHE (2006 a). Reference conditions and ecological status class limits for the indexes according to each river type. CR: reference conditions. V: limit values. EQR: ecological quality ratio. Puntos: sampling points included in the river type (see Table 1). Values of the IBMWP Index were calculated according to reference values from the BOE (2008), values showed as IBMWP(1) and reference values for the other index are those used in CHE (2006 a).

\begin{tabular}{|c|c|c|c|c|c|c|c|c|c|c|c|c|}
\hline Tipo & & & & & 1. Ríos & montañ & nediter & ea silí & & & & \\
\hline Índice & & & & & & & & & & & $I B M$ & \\
\hline CR & & & & & & & & & & & & \\
\hline Clases & V & EQR & V & EQR & V & EQR & V & EQR & V & EQR & $V$ & $E Q R$ \\
\hline B-MB & 140.4 & 0.78 & 5.59 & 0.96 & 24.87 & 0.86 & 1.87 & 0.62 & 12.62 & 0.70 & 142.3 & 0.78 \\
\hline Мo-B & 106.2 & 0.59 & 4.19 & 0.72 & 18.65 & 0.65 & 1.40 & 0.47 & 9.45 & 0.52 & 106.7 & 0.59 \\
\hline D-Mo & 70.2 & 0.39 & 2.80 & 0.48 & 12.44 & 0.43 & 0.93 & 0.31 & 6.30 & 0.35 & 71.1 & 0.39 \\
\hline Ma-D & 36.0 & 0.20 & 1.40 & 0.24 & 6.22 & 0.22 & 0.47 & 0.17 & 3.15 & 0.18 & 35.6 & 0.2 \\
\hline
\end{tabular}

Puntos: 0, A, 1

\begin{tabular}{|c|c|c|c|c|c|c|c|c|c|c|c|c|}
\hline \multirow{2}{*}{$\begin{array}{l}\text { Tipo } \\
\text { Índice }\end{array}$} & \multicolumn{12}{|c|}{ 26. Ríos de montaña húmeda calcárea } \\
\hline & \multicolumn{2}{|c|}{ IBMWP } & \multicolumn{2}{|c|}{ IASPT } & \multicolumn{2}{|c|}{ NFAM } & \multicolumn{2}{|c|}{ NFPLE } & \multicolumn{2}{|c|}{ EPT } & \multicolumn{2}{|c|}{$\operatorname{IBMWP}(1)$} \\
\hline $\mathrm{CR}$ & \multicolumn{2}{|c|}{161} & \multicolumn{2}{|c|}{5.71} & \multicolumn{2}{|c|}{29} & \multicolumn{2}{|c|}{2} & \multicolumn{2}{|c|}{12.3} & \multicolumn{2}{|c|}{147} \\
\hline Clases & $\mathrm{V}$ & EQR & $\mathrm{V}$ & EQR & V & EQR & V & EQR & V & EQR & $V$ & $E Q R$ \\
\hline $\mathrm{B}-\mathrm{MB}$ & 127.2 & 0.79 & 5.07 & 0.89 & 25.27 & 0.87 & 1 & 0.5 & 8.27 & 0.67 & 116 & 0.79 \\
\hline Mo-B & 95.0 & 0.59 & 3.80 & 0.67 & 18.95 & 0.65 & 0.75 & 0.38 & 6.20 & 0.50 & 87 & 0.59 \\
\hline D-Mo & 62.8 & 0.39 & 2.54 & 0.44 & 12.64 & 0.44 & 0.50 & 0.25 & 4.13 & 0.34 & 58 & 0.39 \\
\hline Ma-D & 32.2 & 0.20 & 1.27 & 0.224 & 6.32 & 0.22 & 0.25 & 0.12 & 2.07 & 0.17 & 29 & 0.20 \\
\hline
\end{tabular}

Puntos: 2

\begin{tabular}{|c|c|c|c|c|c|c|c|c|c|c|c|c|}
\hline \multirow{2}{*}{$\begin{array}{c}\text { Tipo } \\
\text { Índice }\end{array}$} & \multicolumn{12}{|c|}{ 12. Ríos de montaña mediterránea calcárea } \\
\hline & \multicolumn{2}{|c|}{ IBMWP } & \multicolumn{2}{|c|}{ IASPT } & \multicolumn{2}{|c|}{ NFAM } & \multicolumn{2}{|c|}{ NFPLE } & \multicolumn{2}{|c|}{ EPT } & \multicolumn{2}{|c|}{$\operatorname{IBMWP}(1)$} \\
\hline $\mathrm{CR}$ & \multicolumn{2}{|c|}{150} & \multicolumn{2}{|c|}{4.89} & \multicolumn{2}{|c|}{25.3} & \multicolumn{2}{|c|}{0.91} & \multicolumn{2}{|c|}{6.2} & \multicolumn{2}{|c|}{126} \\
\hline Clases & $\mathrm{V}$ & EQR & V & EQR & V & EQR & V & EQR & V & EQR & $V$ & $E Q R$ \\
\hline $\mathrm{B}-\mathrm{MB}$ & 133.5 & 0.89 & 4.79 & 0.98 & 19.35 & 0.76 & 0.42 & 0.46 & 5.48 & 0.88 & 105.75 & 0.88 \\
\hline Mo-B & 100.5 & 0.67 & 3.59 & 0.73 & 14.51 & 0.57 & 0.32 & 0.35 & 4.11 & 0.66 & 79.31 & 0.66 \\
\hline D-Mo & 67.5 & 0.45 & 2.40 & 0.49 & 9.68 & 0.38 & 0.21 & 0.23 & 2.74 & 0.44 & 52.87 & 0.44 \\
\hline Ma-D & 33.0 & 0.22 & 1.20 & 0.25 & 4.84 & 0.19 & 0.11 & 0.12 & 1.37 & 0.20 & 26.44 & 0.22 \\
\hline
\end{tabular}

Puntos: 3, 4, 5 
recopiló información de los estudios de la CHE publicados (CHE, 2004, 2007), así como la relación de presiones e impactos de la cuenca, incluidas en el Plan Hidrológico de la cuenca del
Oja-Tirón (Confederación Hidrográfica del Ebro, Junta de Castilla y León y Gobierno de La Rioja, 2007), con el fin de comprobar el grado de aplicación de la DMA.

Tabla 3. Taxones encontrados en cada punto de muestreo para el período 2003-07. 0: Llanos de la Casa, Oja, A: Llanos de la Casa, afluente, 1: Posadas, 2: Ezcaray, 3: Santurde, 4: Santo Domingo de la Calzada, 5: Casalarreina. Taxa found at each sampling point for the 2003-07 period. 0: Llanos de la Casa, Oja River, A: Llanos de la Casa, tributary, 1: Posadas, 2: Ezcaray, 3: Santurde, 4: Santo Domingo de la Calzada, 5: Casalarreina.

\begin{tabular}{|c|c|c|c|c|c|c|c|c|c|c|c|c|c|c|c|}
\hline Taxón & A & 0 & 1 & 2 & 3 & 4 & 5 & Taxón (cont) & $\mathrm{A}$ & 0 & 1 & 2 & 3 & 4 & 5 \\
\hline TRICLADIDA & & & & & & & & Elmidae & + & + & + & + & + & + & + \\
\hline Planariidae & & & + & + & & & + & Haliplidae & & & + & + & + & & + \\
\hline OLIGOCHAETA & + & + & + & + & + & + & + & Helodidae & + & + & & & & & \\
\hline HIRUDINEA & & & & & & & & Hydraenidae & + & + & + & + & & + & + \\
\hline Erpobdellidae & & & + & + & & & + & Hydrophilidae & & & & + & & & + \\
\hline Glossiphoniidae & & & + & + & + & & + & TRICHOPTERA & & & & & & & \\
\hline MOLLUSCA & & & & & & & & Beraeidae & & & + & & & & \\
\hline Ancylidae & + & + & + & + & + & + & + & Brachycentridae & + & + & + & + & & & + \\
\hline Hydrobiidae & & & & + & & & + & Glossosomatidae & + & + & + & + & & & \\
\hline Lymnaeidae & & & & + & + & + & + & Goeridae & + & + & + & + & & & \\
\hline Physidae & & & & & & & + & Hydropsychidae & + & + & + & + & & + & + \\
\hline Planorbidae & & & & + & & + & + & Hydroptilidae & & + & + & + & + & + & + \\
\hline Sphaeriidae & & & & + & & & + & Lepidostomatidae & + & + & + & & & & + \\
\hline HYDRACARINA & + & + & + & + & + & + & + & Leptoceridae & & + & & & & & + \\
\hline OSTRACODA & + & + & + & + & + & + & + & Limnephilidae & + & + & + & + & + & & \\
\hline AMPHIPODA & & & & & & & & Odontoceridae & + & + & + & & & & \\
\hline Gammaridae & & & & + & + & & + & Philopotamidae & + & + & + & & & & \\
\hline ISOPODA & & & & & & & & Polycentropodidae & & + & + & + & & & + \\
\hline Asellidae & & & + & + & + & + & + & Psychomyiidae & & & + & + & & & + \\
\hline EPHEMEROPTERA & & & & & & & & Rhyacophilidae & + & + & + & + & & & + \\
\hline Baetidae & + & + & + & + & + & + & + & Sericostomatidae & + & + & + & + & & & + \\
\hline Caenidae & & & + & + & + & + & + & Uenoidae & & + & + & & & & \\
\hline Ephemerellidae & + & + & + & + & + & + & + & LEPIDOPTERA & & & & & & & \\
\hline Heptageniidae & + & + & + & + & + & + & + & Pyralidae & & & & + & & + & \\
\hline Leptophlebiidae & + & + & + & + & + & & + & DIPTERA & & & & & & & \\
\hline Potamanthidae & & & & & & & + & Athericidae & + & + & + & + & & & + \\
\hline ODONATA & & & & & & & & Blephariceridae & + & + & + & & & & \\
\hline Coenagrionidae & & & & & & & + & Ceratopogonidae & + & + & + & + & + & + & + \\
\hline PLECOPTERA & & & & & & & & Chironomidae & + & + & + & + & + & + & + \\
\hline Capniidae & & & + & + & + & + & & Culicidae & & & & + & & & \\
\hline Chloroperlidae & + & + & + & + & + & & & Dixidae & & & + & & & & + \\
\hline Leuctridae & + & + & + & + & + & + & + & Dolichopodidae & & & + & + & & + & \\
\hline Nemouridae & + & + & + & + & + & + & + & Empididae & + & + & + & + & + & + & + \\
\hline Perlidae & + & + & + & + & & & & Ephydridae & & & & + & + & + & + \\
\hline Perlodidae & + & + & + & + & + & + & + & Limoniidae & + & + & + & + & + & + & + \\
\hline Taeniopterygidae & + & + & + & + & & + & & Muscidae & & & & + & + & + & + \\
\hline HETEROPTERA & & & & & & & & Psychodidae & + & + & + & + & + & + & + \\
\hline Corixidae & & & & & & + & + & Rhagionidae & + & + & + & + & & + & + \\
\hline Hydrometridae & & & & & & & + & Simuliidae & + & + & + & + & + & + & + \\
\hline NEUROPTERA & & & & & & & & Stratiomyidae & & & + & & & + & \\
\hline Sialidae & & & + & & & & + & Syrphidae & & & & & + & + & \\
\hline COLEOPTERA & & & & & & & & Tabanidae & & & & + & + & & + \\
\hline Dryopidae & & & & & & & + & Thaumaleidae & & & & + & & & \\
\hline Dytiscidae & & & + & + & + & + & + & Tipulidae & & & + & + & & + & + \\
\hline
\end{tabular}




\section{RESULTADOS}

\section{Estado ecológico del río Oja durante el periodo 2003-2007}

Se han encontrado 77 taxones de macroinvertebrados, 74 de ellos incluidos en el IBMWP, (Tabla 3). El NFAM oscila entre las 9 del punto 4 (febrero de 2004) y las 40 del punto 2 (agosto de 2003) (Tabla 4). Hay familias con representantes en todos los tramos (Ancylidae, Baetidae, Ephemerellidae, Heptageniidae, Leuctridae, Nemouri- dae, Perlodidae, Dytiscidae, Elmidae, Hydroptilidae, Ceratopogonidae, Chironomidae, Empididae, Limoniidae, Psychodidae, Simuliidae, Oligochaeta, Hidracarina, Ostracoda), mientras que otras aparecen únicamente en cabecera (p.e. Perlidae, Brachycentridae, Glossosomatidae, Goeridae, Uenoidae) o en tramos medios y bajos (p.e. Physidae, Planorbidae, Sphaeridae, Gammaridae, Corixidae, Syrphidae, Tabanidae).

La Tabla 4 resume los valores de los diferentes índices (por tramo y fecha) y de sus correspondientes ratios de estado ecológico (EQR), asig-

Tabla 4. Valores obtenidos para los distintos índices aplicados. P: Punto de muestreo (ver Tabla 1). V: Valor del índice, EQR: Ratio de estado ecológico, C: clase de calidad del agua. Las condiciones de referencia utilizadas son las de la Tabla 2. Values obtained for different index applied. P: Sampling point (see Table 1). V: Index value, EQR: Ecological Quality Ratio, C: water quality class. The reference conditions used are those from Table 2.

\begin{tabular}{|c|c|c|c|c|c|c|c|c|c|c|c|c|c|c|c|c|}
\hline \multirow[b]{2}{*}{ Fecha } & \multirow[b]{2}{*}{$\mathrm{P}$} & \multicolumn{3}{|c|}{ IBMWP } & \multicolumn{3}{|c|}{ IASPT } & \multicolumn{3}{|c|}{ NFAM } & \multicolumn{3}{|c|}{ NFPLE } & \multicolumn{3}{|c|}{ EPT } \\
\hline & & V & EQR & $\mathrm{C}$ & V & EQR & $\mathrm{C}$ & V & EQR & $\mathrm{C}$ & V & EQR & $\mathrm{C}$ & V & EQR & $\mathrm{C}$ \\
\hline \multirow{5}{*}{ May 03} & 1 & 200 & 1.11 & MB & 6.67 & 1.17 & $\mathrm{MB}$ & 30 & 1.04 & MB & 5 & 1.7 & $\mathrm{MB}$ & 16 & 0.89 & $\mathrm{MB}$ \\
\hline & 2 & 143 & 0.89 & $\mathrm{MB}$ & 5.5 & 0.96 & $\mathrm{MB}$ & 26 & 0.90 & $\mathrm{MB}$ & 4 & 2.0 & $\mathrm{MB}$ & 11 & 0.89 & $\mathrm{MB}$ \\
\hline & 3 & 136 & 0.91 & $\mathrm{MB}$ & 5.23 & 1.07 & $\mathrm{MB}$ & 26 & 1.03 & MB & 5 & 5.5 & $\mathrm{MB}$ & 10 & 1.61 & $\mathrm{MB}$ \\
\hline & 4 & 123 & 0.82 & B & 5.12 & 1.05 & $\mathrm{MB}$ & 24 & 0.95 & $\mathrm{MB}$ & 4 & 4.4 & $\mathrm{MB}$ & 9 & 1.45 & $\mathrm{MB}$ \\
\hline & 5 & 92 & 0.61 & Mo & 5.11 & 1.05 & $\mathrm{MB}$ & 18 & 0.71 & B & 1 & 1.1 & $\mathrm{MB}$ & 6 & 0.97 & $\mathrm{MB}$ \\
\hline \multirow{3}{*}{ Ago 03} & 1 & 215 & 1.19 & $\mathrm{MB}$ & 6.32 & 1.08 & $\mathrm{MB}$ & 34 & 1.18 & $\mathrm{MB}$ & 5 & 1.7 & $\mathrm{MB}$ & 16 & 0.89 & $\mathrm{MB}$ \\
\hline & 2 & 197 & 1.22 & MB & 4.93 & 0.86 & B & 40 & 1.38 & MB & 2 & 1.0 & $\mathrm{MB}$ & 13 & 1.06 & $\mathrm{MB}$ \\
\hline & 5 & 150 & 1.00 & MB & 5.00 & 1.02 & $\mathrm{MB}$ & 30 & 1.19 & MB & 2 & 2.2 & $\mathrm{MB}$ & 10 & 1.61 & MB \\
\hline \multirow{5}{*}{ Nov 03} & 1 & 193 & 1.07 & $\mathrm{MB}$ & 6.66 & 1.14 & $\mathrm{MB}$ & 29 & 1.01 & $\mathrm{MB}$ & 7 & 2.3 & $\mathrm{MB}$ & 17 & 0.94 & $\mathrm{MB}$ \\
\hline & 2 & 148 & 0.92 & $\mathrm{MB}$ & 5.29 & 0.93 & $\mathrm{MB}$ & 28 & 0.97 & $\mathrm{MB}$ & 5 & 2.5 & $\mathrm{MB}$ & 11 & 0.89 & $\mathrm{MB}$ \\
\hline & 3 & 67 & 0.45 & D & 4.19 & 0.86 & B & 16 & 0.63 & B & 1 & 1.1 & $\mathrm{MB}$ & 4 & 0.65 & B \\
\hline & 4 & 64 & 0.43 & D & 4.00 & 0.82 & B & 16 & 0.63 & B & 1 & 1.1 & $\mathrm{MB}$ & 4 & 0.65 & B \\
\hline & 5 & 176 & 1.17 & MB & 5.33 & 1.09 & $\mathrm{MB}$ & 33 & 1.30 & MB & 0 & 0 & $\mathbf{M}$ & 12 & 1.94 & $\mathrm{MB}$ \\
\hline \multirow{5}{*}{ Feb 04} & 1 & 231 & 1.28 & $\mathrm{MB}$ & 6.79 & 1.17 & $\mathrm{MB}$ & 34 & 1.18 & $\mathrm{MB}$ & 6 & 2.0 & $\mathrm{MB}$ & 19 & 1.06 & $\mathrm{MB}$ \\
\hline & 2 & 126 & 0.78 & B & 5.48 & 0.96 & $\mathrm{MB}$ & 23 & 0.79 & B & 4 & 2.0 & $\mathrm{MB}$ & 10 & 0.81 & $\mathrm{MB}$ \\
\hline & 3 & 92 & 0.61 & Mo & 5.11 & 1.05 & $\mathrm{MB}$ & 18 & 0.71 & B & 3 & 3.3 & $\mathrm{MB}$ & 7 & 1.13 & $\mathrm{MB}$ \\
\hline & 4 & 45 & 0.30 & D & 5.00 & 1.02 & $\mathrm{MB}$ & 9 & 0.36 & D & 3 & 3.3 & $\mathrm{MB}$ & 4 & 0.65 & B \\
\hline & 5 & 127 & 0.85 & B & 4.88 & 1.00 & MB & 26 & 1.03 & MB & 2 & 2.2 & $\mathrm{MB}$ & 10 & 1.61 & MB \\
\hline Jun 04 & 1 & 217 & 1.21 & $\mathrm{MB}$ & 6.58 & 1.13 & $\mathrm{MB}$ & 33 & 1.15 & $\mathrm{MB}$ & 3 & 1.0 & $\mathrm{MB}$ & 16 & 0.89 & $\mathrm{MB}$ \\
\hline Nov 04 & 5 & 131 & 0.87 & B & 5.46 & 1.12 & $\mathrm{MB}$ & 24 & 0.95 & $\mathrm{MB}$ & 0 & 0 & $\mathbf{M}$ & 10 & 1.61 & B \\
\hline Mar 05 & 5 & 138 & 0.92 & $\mathrm{MB}$ & 5.75 & 1.18 & $\mathrm{MB}$ & 24 & 0.95 & $\mathrm{MB}$ & 2 & 2.2 & $\mathrm{MB}$ & 12 & 1.94 & $\mathrm{MB}$ \\
\hline \multirow{2}{*}{ May 05} & 0 & 208 & 1.16 & MB & 6.71 & 1.15 & $\mathrm{MB}$ & 31 & 1.08 & MB & 5 & 1.7 & $\mathrm{MB}$ & 17 & 0.94 & $\mathrm{MB}$ \\
\hline & A & 202 & 1.12 & MB & 6.31 & 1.08 & MB & 32 & 1.11 & MB & 6 & 2.0 & $\mathrm{MB}$ & 16 & 0.89 & MB \\
\hline \multirow{3}{*}{ Ago 05} & 0 & 95 & 0.53 & Mo & 6.33 & 1.09 & MB & 15 & 0.52 & Mo & 1 & 0.3 & Mo & 9 & 0.50 & B \\
\hline & A & 129 & 0.72 & B & 7.59 & 1.30 & $\mathrm{MB}$ & 17 & 0.59 & Mo & 3 & 1.0 & $\mathrm{MB}$ & 14 & 0.78 & $\mathrm{MB}$ \\
\hline & 1 & 105 & 0.58 & Mo & 6.18 & 1.06 & $\mathrm{MB}$ & 17 & 0.59 & Mo & 1 & 0.3 & Mo & 9 & 0.50 & Mo \\
\hline \multirow{2}{*}{ Jun 06} & 1 & 176 & 0.98 & $\mathrm{MB}$ & 6.52 & 1.12 & $\mathrm{MB}$ & 27 & 0.94 & MB & 4 & 1.3 & MB & 16 & 0.89 & $\mathrm{MB}$ \\
\hline & 5 & 118 & 0.79 & B & 4.92 & 1.01 & MB & 24 & 0.95 & $\mathrm{MB}$ & 1 & 1.1 & $\mathrm{MB}$ & 9 & 1.45 & MB \\
\hline Jun 07 & 5 & 149 & 0.99 & MB & 4.66 & 0.95 & B & 32 & 1.26 & MB & 1 & 1.1 & $\mathrm{MB}$ & 9 & 1.45 & $\mathrm{MB}$ \\
\hline
\end{tabular}


nando las clases de calidad de acuerdo a los límites de clases de calidad incluidos en la Tabla 2.

En conjunto, para el IBMWP el $75.86 \%$ de las medidas dan un estado Muy Bueno-Bueno, con un $10.35 \%$ de calidad Deficiente. Esta deficiencia aparece en dos puntos del ecotipo 12 (3 y 4), y probablemente se deba a la irregularidad del caudal del río en este tramo que sufre sequía desde finales de primavera hasta principios de otoño.

En cuanto al resto de los índices, el estado Muy Bueno-Bueno aparece en el $100 \%$ de los valores del IASPT, en el $86.21 \%$ del NFAM y del NFPLE y en el $96.55 \%$ del EPT, con un $6.9 \%$ de valores Malos en el NFPLE, un $3.45 \%$ de valores Deficientes en el NFAM y un $3.45 \%$ de valores Moderados en el EPT.

La figura 2 representa las clases de calidad encontradas para los distintos índices y puntos de muestreo. El ecotipo 26, que se corresponde con la estación R-2 (Ezcaray), presenta para todos los índices un estado Muy Bueno-Bueno.

En lo referente al IBMWP, el ecotipo 11 (puntos A, 0 y 1) presenta un estado Muy BuenoBueno en el $81.82 \%$ de los casos, mientras que el ecotipo 12 (puntos 3 a 5) tiene un $64.29 \%$ de los valores en la incluidos en la clase Muу BuenaBuena. En este tramo el punto 4 (Santo Domingo) es el que presenta el peor estado de todos.

En cuanto al resto de los índices, los valores del IASPT están todos incluidos en la clase de calidad Muy Buena-Buena. El NFAM presenta valores de las clases Muy Buena y Moderada en el ecotipo 11, y Muy Buena-Buena en el ecotipo 12, exceptuando el punto 4 , donde también aparece la clase Deficiente. El NFPLE presenta valores de calidad Muy Buena, excepto en los puntos 0 y 1, con un estado Moderado y en el punto 5, con un valor Malo. Para finalizar, el EPT presenta un estado Muy Bueno y Moderado en el ecotipo 11, mientras que en el 12 el estado es Muy BuenoBueno. Se observa que los peores valores de los distintos índices aparecen en verano en el ecotipo 11 (agosto 2005), mientras que en el ecotipo 12 se corresponden con los datos de noviembre de 2003 y febrero de 2004, cuando se ha recuperado el agua en estos tramos de río (puntos 3 y 4).

La aparente disparidad entre los estados de las masas de agua indicados por el IBMWP y los reflejados por el resto de los índices probablemente se deba a la reciente modificación de los valores de referencia del IBMWP (BOE, 2008), que no se ha aplicado al resto de los índices (no han sido incluidos en la normativa) por lo que sería recomendable revisar también los valores de referencia establecidos para el resto de los índices biológicos utilizados.

Los únicos valores de calidad Mala en el río Oja aparecen en el punto 5 (Casalarreina) para el índice NFPLE en el mes de noviembre, debido a la inexistencia de Plecópteros.

\section{Evolución de la calidad del agua en el río Oja (periodo 1994-2007)}

Para estudiar la evolución de la calidad del agua se han utilizado los valores de IBMWP de los informes existentes (CHE, 2004, incluye datos del periodo 1994-2004; CHE, 2006) así como datos propios (periodo 2003-2007).

Los límites entre las distintas clases de calidad para el IBMWP fueron establecidos originariamente por Alba-Tercedor y Sánchez Ortega (1988) y durante los últimos 20 años se han ido modificando, adaptándose p.e. a tipos generales de río, comosilíceos o calcáreos de cabecera (AlbaTercedor et al., 2002), ecorregiones (CHE, 2004) o ecotipos (MIMAN, 2005), siendo estos últimos los elegidos para delimitar las condiciones de referencia en la normativa más reciente (BOE, 2008).

Con el fin de evaluar la evolución de la calidad del agua en los distintos años, se han utilizado las condiciones de referencia del BOE de 2008 para el cálculo de los límites entre clases de calidad. Debido a esto, parte de los datos históricos aparecen incluidos en una clase diferente a la que fueron asignados en su momento.

La figura 3 representa los valores del IBMWP para el período 1994-2007, provenientes de los informes de la CHE (triángulos) y de datos propios (cuadrados). De los cuatro puntos incluidos en los informes, tres coinciden con los muestreados por nuestro equipo (Ezcaray, Santo Domingo y Casalarreina), mientras que el cuarto (Azarrulla) se sitúa aguas abajo de nuestro punto de muestreo (Posadas). Estos dos últimos puntos (ecotipo 11) se han agrupado en un solo gráfico, 


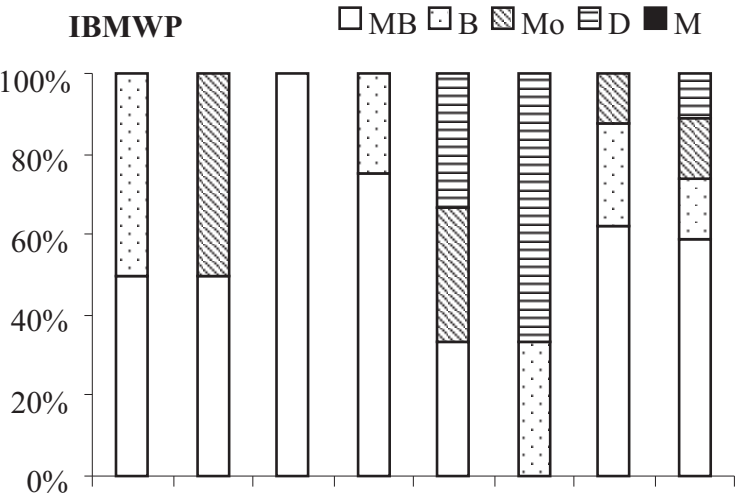

$\begin{array}{llllllll}\text { A } & 0 & 1 & 2 & 3 & 4 & 5 & \text { G }\end{array}$
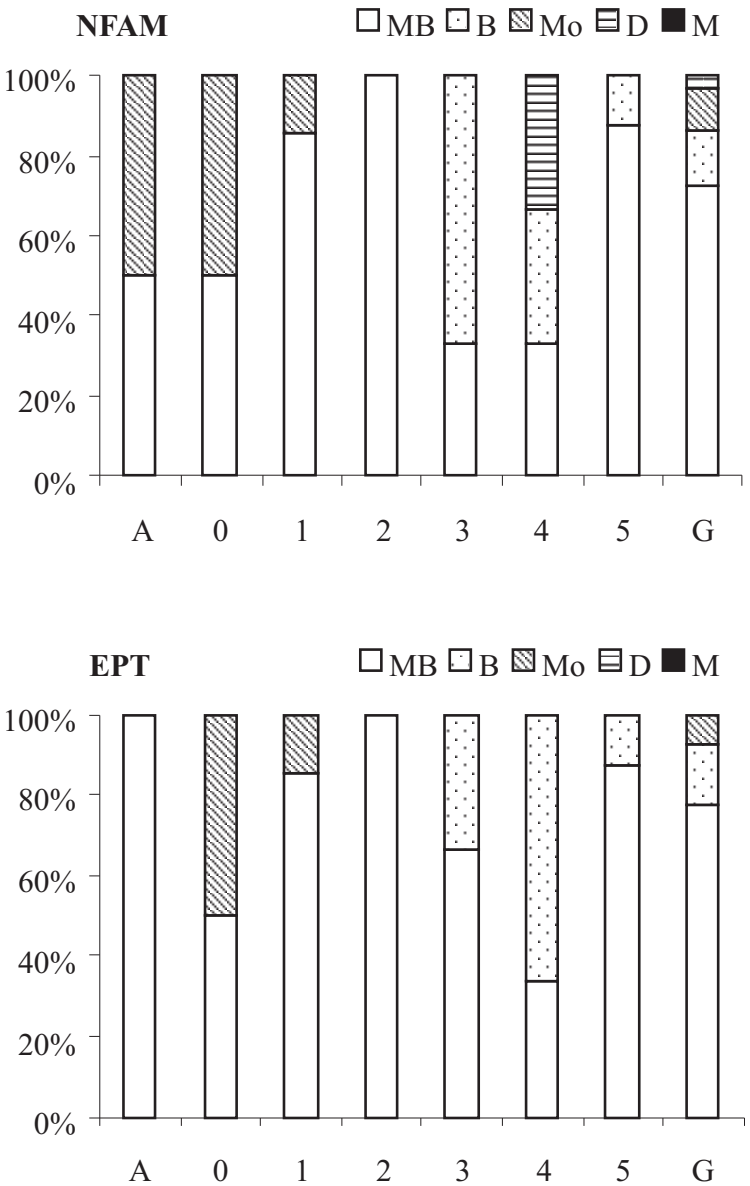

IASPT $\square \mathrm{MB} \square \mathrm{B} \mathbf{S}_{\mathrm{Mo}} \Xi_{\mathrm{D}} \square_{\mathrm{M}}$

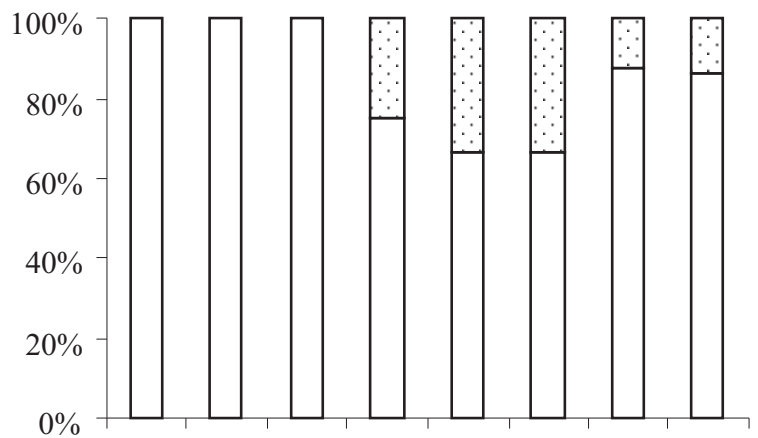

$\begin{array}{llllllll}\text { A } & 0 & 1 & 2 & 3 & 4 & 5 & \text { G }\end{array}$

NFPLE $\square \mathrm{MB} \square \mathrm{B}$ 㐁

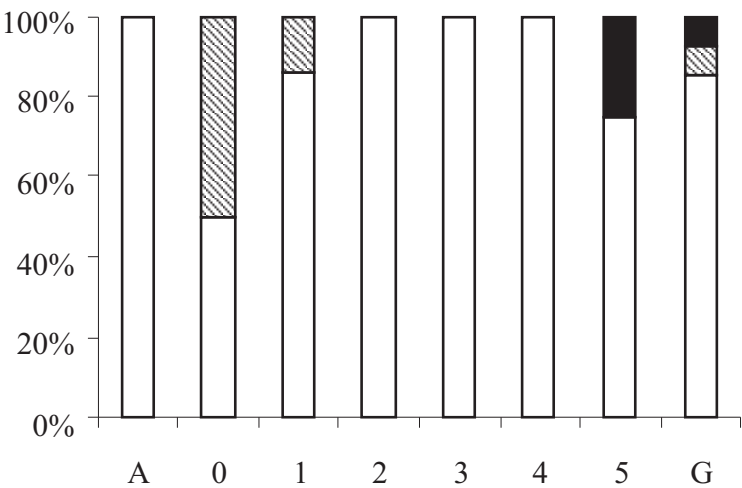

IBMWP: Iberian Biomonitoring Working Party

IASPT: Iberian Average Score per Taxon

NFAM: Número de familias

NFPLE: Número de familias de Plecópteros

EPT: Número de familias de Efemerópteros, Plecópteros y Tricópteros

Figura 2. Importancia relativa de los distintos estados de calidad del agua (en \%) en cada punto y en conjunto (G) para cada índice biológico (años 2003-2007). $\mathrm{N}^{\circ}$ muestras: 29. Relative importance of the different water quality states (in \%) in each point and all together $(G)$ for each biological index (years 2003-2007). number of samples: 29.

donde los triángulos representan los valores de Azarrulla y los cuadrados los de Posadas.
Se observa que en todos los puntos excepto Santo Domingo, los valores de IBMWP han ido incre- 

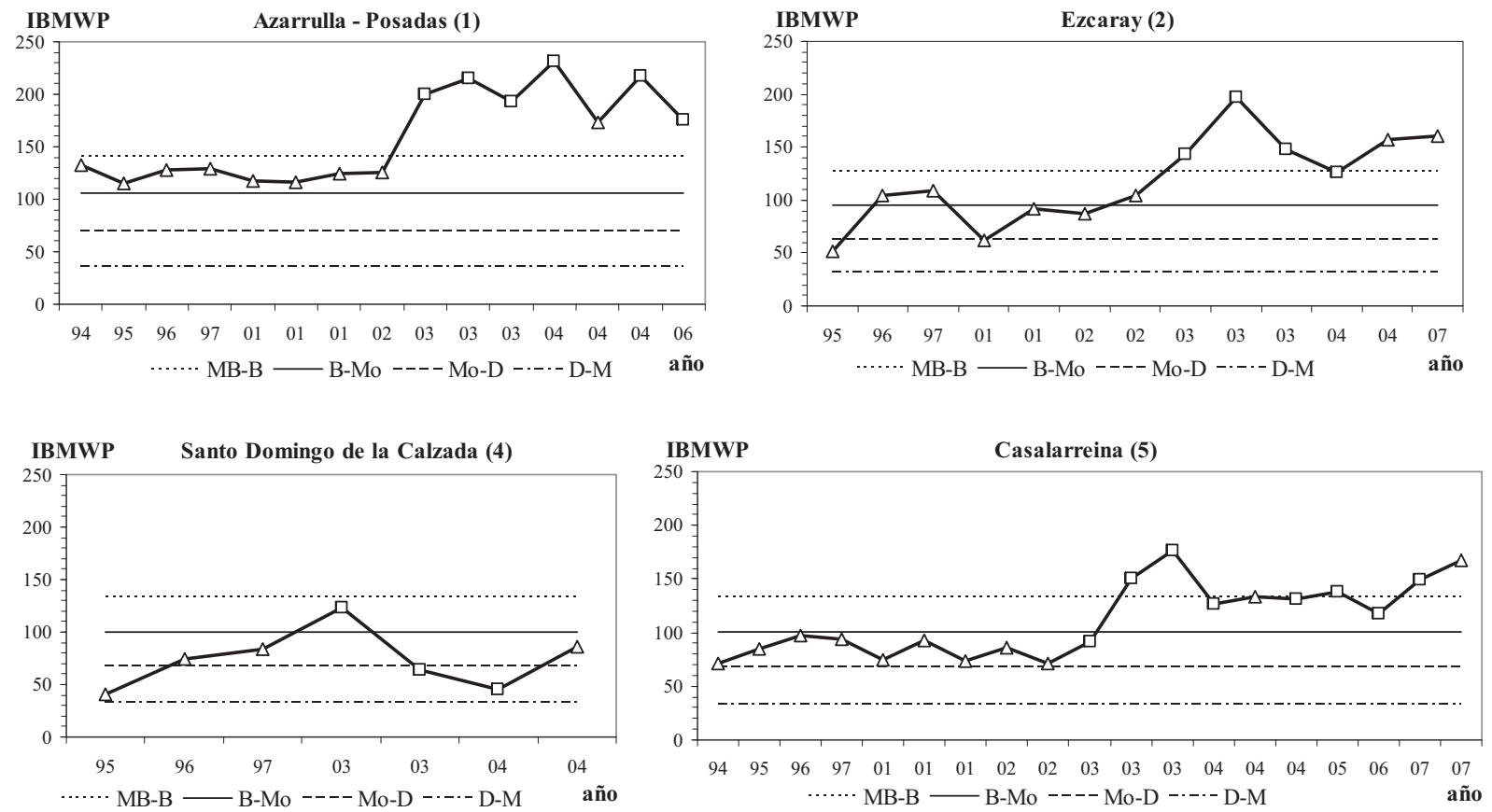

Figura 3. Evolución de la calidad del agua del río Oja (valores del IBMWP) para el periodo 1994-2007, según el punto de muestreo. Triángulos: datos de los informes de la CHE (2004, 2007). Cuadrados: datos propios. Las líneas horizontales representan los valores límite entre clases, según el BOE (2008). Fechas (por orden): 94 = octubre; 95 = marzo; 96 = mayo; 97 = junio; 01 = junio, julio; 02 = agosto; 03 = mayo, agosto, noviembre; 04 = febrero, marzo (CHE, 2004), junio, noviembre; $05=$ marzo; $06=$ junio; $07=$ junio, julio (CHE, 2007). Evolution of the Oja River water quality (IBMWP values) for the years 1994-2006, according to sampling point. Triangles: data from CHE reports (2004, 2007). Squares: own data. Horizontal lines represent the limit values between classes, following BOE (2008). Dates (in order): 94 = October; $95=$ March; $96=$ May; $97=$ June; $01=$ June, July; $02=$ August; $03=$ May, August, November; $04=$ February, March (CHE, 2004), June, November; $05=$ March; $06=$ June; $07=$ June, July (CHE, 2007).

mentándose a lo largo del tiempo. En AzarrullaPosadas los datos históricos (1994-2002) presentan un estado Bueno (Muy Bueno en la clasificación original) mientras que los datos más recientes (2003-2006) están en estado Muy Bueno. En Ezcaray el primer dato histórico presenta un estado Deficiente (clasificado previamente como Moderado), mejorando en años posteriores hasta un estado Moderado a Bueno (Bueno o Muy Bueno originariamente), у Muy Bueno (datos 2003-2006). En cuanto a Casalarreina, los datos históricos (1994-2002) muestran un estado Moderado (Bueno previamente), que sube hasta Muy Bueno y Bueno en los últimos muestreos. El primer muestreo de nuestro equipo (mayo 2003) es el último que aparece con calidad Moderada.

En Santo Domingo de la Calzada, como ya hemos comentado anteriormente, el río se seca aproximadamente desde el final de la primave- ra hasta el principio de otoño-invierno, variando su temporalidad según el año. Por este motivo solo se dispone de valores que corresponden a los meses con agua ( 7 muestras). De ellos, 5 se corresponderían a calidades Buena o Muy Buena según los valores de referencia originales, pero según los parámetros actuales sólo uno (mayo 2003) quedaría con calidad Buena y el resto bajaría a calidad Moderada. En las muestras de noviembre de 2003 y febrero de 2004 la calidad aparece como Deficiente, debido probablemente a las obras de acondicionamiento en el cauce en el tramo cercano a la ciudad, que generaron gran cantidad de materiales en suspensión, afectando al ecosistema acuático que esos momentos se estaba recuperando de la sequía. Este hecho viene corroborado por la subida a calidad Modera$d a$ el mes siguiente a nuestro muestreo (Fig. 3). Podemos resumir diciendo que en este punto se 
observa un aumento de los valores del IBMWP de manera cíclica, desde la recuperación del caudal en otoño-invierno hasta su desaparición en primavera-verano, lo que le permitiría cumplir con los objetivos de la DMA al menos en los meses de primavera. Nuestros datos más recientes (primavera-verano 2008 y 2009, en preparación) corroboran este hecho, al alcanzar este punto un estado Muy Bueno o Bueno en estos meses.

\section{DISCUSIÓN}

El incremento de la población en las regiones de clima mediterráneo origina una competencia por los recursos hídricos que limita la capacidad de los ríos para sobrevivir como sistemas sostenibles y autorregulados (Gasith \& Resh, 1999). De manera similar a otros ríos mediterráneos (p.e. Gallardo-Mayenco et al., 2004), en el Oja las mayores presiones sobre los ecosistemas acuáticos son la falta de caudal y los contaminantes, sobre todo orgánicos, siendo estos últimos los principales reguladores de las poblaciones. La reducción de caudal durante el verano afectaría tanto a los tramos altos, que ven reducidas sus comunidades, como a los tramos medios, donde desaparecen totalmente al secarse totalmente el río. En este caso la recuperación del agua en otoño equivaldría a la crecida de primavera, originando una comunidad invernal poco diversa y abundante (Gasith \& Resh, 1999).

Tomando como valores de referencia para los distintos estados de las masas de agua los referidos en el BOE de 2008, se ha observado una mejora generalizada de la calidad del agua en los últimos años en tres de los puntos de muestreo. En la figura 3 se observa un incremento de los valores del IBMWP a partir del año 2002 en Azarrulla-Posadas, Ezcaray y Casalarreina, cuando se pusieron en marcha las EDAR de Ezcaray (septiembre 2002) y de Haro (mayo 2002), esta última con un colector que recoge las aguas de los principales núcleos urbanos del valle del Oja por debajo de Ezcaray. En 2002-2003 se han puesto además en marcha otros sistemas en poblaciones de cabecera con menos de 100 habitantes, como fosas sépticas y decantadores. Estas infraestruc- turas han evitado los vertidos directos al río de los principales núcleos urbanos, disminuyendo la carga orgánica y mejorando la calidad del agua. En los planes de saneamiento previstos para los próximos años se incluyen la construcción de nuevas depuradoras, la conexión de nuevos núcleos de población a las depuradores existentes y la ampliación de los tratamientos de algunas de ellas, lo que teóricamente debería traducirse en un incremento de la calidad aguas abajo. En nuestros datos más recientes $(2008,2009$, en preparación) se observa que el estado ecológico de todos los tramos presenta valores Muy Buenos-Buenos en los puntos 1, 2, 4 y 5 para el IBMWP, por lo que podríamos concluir que en este aspecto (calidad biológica) se podría alcanzar el objetivo de la DMA.

Por otro lado, en Casalarreina, aunque los últimos informes de índices bióticos señalan una calidad Buena o Muy Buena (CHE, 2007, 2008), la elevada concentración de nitratos que proviene del acuífero subyacente $\left(20.5 \mathrm{mg} / \mathrm{l} \mathrm{NO}_{3}\right.$ en $2007 ; 37.30 \mathrm{mg} / \mathrm{l} \mathrm{NO}_{3}$ en 2008) hace que la calidad final baje hasta Moderada (límite B-Mo: $20 \mathrm{mg} / \mathrm{NO}_{3}$ ). Se han publicado recientemente trabajos sobre la caracterización hidroquímica (Martínez-Bastida et al., 2007) o la dinámica del agua y sus contaminantes entre la zona saturada y no saturada (Arauzo et al., 2006, 2007, 2010; Martínez-Bastida et al., 2009 a, b) y en estos momentos se está haciendo un seguimiento de la dinámica a escala horaria del agua de drenaje en el suelo (Arauzo et al., 2010), aspecto muy importante a la hora de hacer un uso racional tanto del agua de regadío como de los fertilizantes, que permitiría por una parte un ahorro monetario para el agricultor, y por otra ayudaría a disminuir el nitrógeno lixiviado en el acuífero. Este aspecto (calidad química del agua) sería el que presentaría mayor dificultad para alcanzar los objetivos de la DMA, ya que se necesitaría tanto un estudio a nivel de cuenca de las interacciones suelos-acuífero-río, que permitiera un conocimiento más profundo de la dinámica del agua, así como un cambio de mentalidad de los agricultores de la zona, promoviendo la aplicación de buenas prácticas agrícolas, con el fin de reducir los nitratos presentes en el acuífero y por tanto, en los tramos bajos del río. 
Por último, la existencia de periodos de sequía está muy ligada a la presión extractiva de agua para abastecimiento y regadío, siendo esta última la cuota más importante en la cuenca del OjaTirón: $65.2 \mathrm{hm}^{3}$ totales, de los cuales $4.3 \mathrm{hm}^{3}$ son para abastecimiento, ganadería e industria (GLR, 2007). En estos momentos está proyectada la explotación del acuífero de Pradoluengo-Anguiano (cercano a Ezcaray) para abastecimiento, lo que podría disminuir en un futuro la presión sobre el acuífero del Oja. El aumento hacia cabecera de los tramos que se secan en verano, como ha sucedido en los últimos años, haría bajar la calidad del agua en la zona de Ezcaray, revirtiendo la tendencia actual de mejora que se viene observando.

Para finalizar, queremos incidir en la necesidad de llevar a cabo estudios más profundos a nivel de pequeñas cuencas, con el fin de conocer mejor la dinámica del agua de las mismas y su relación con el entorno, tanto natural como antrópico. Esta información, que acaba diluyéndose cuando se ofrece en un informe global, p.e. a nivel de demarcación hidrográfica, facilitaría la consecución de los objetivos de la DMA previstos para cada una de las zonas que la integran.

\section{AGRADECIMIENTOS}

Queremos agradecer a Antonio y Abel Domínguez, Juan Carlos y Yolanda Fernández y Carmen Gutiérrez su ayuda en alguno de los muestreos que hemos realizado y a Maria Jesús Loyo (Txispi), propietaria de Casa Ugarte (Ojacastro) por su amabilidad y buena compañía. Este trabajo se ha financiado a través de los proyectos del Plan Nacional de I+D REN2002-02550 y AGL 2006-07087/AGR y una Beca de la Comunidad de Madrid (GR/AMB/0745/2004).

\section{BIBLIOGRAFÍA}

ALBA-TERCEDOR, J. \& A. SÁNCHEZ-ORTEGA. 1988. Un método rápido y simple para evaluar la calidad biológica de las aguas corrientes basado en el de Hellawell (1978). Limnetica, 4: 51-56.

ALBA-TERCEDOR, J., P. JÁIMEZ-CUÉLLAR, M.
ÁLVAREZ, J. AVILÉS, N. BONADA, J. CASAS, A. MELLADO, M. ORTEGA, I. PARDO, N. PRAT, M. RIERADEVALL, S. ROBLES, C.E. SÁINZ-CANTERO, A. SÁNCHEZ-ORTEGA, M.L. SUÁREZ, M. TORO, M.R. VIDALABARCA, S. VIVAS \& C. ZAMORA-MUÑOZ, 2002. Caracterización del estado ecológico de ríos mediterráneos ibéricos mediante el índice IBMWP (antes BMWP'). Limnetica, 21(3-4): 175-185.

ARAUZO, M., M. VALLADOLID, J. J. MARTÍNEZ-BASTIDA \& C. GUTIÉRREZ. 2006. Dinámica espacio-temporal del contenido en nitrato de las aguas superficiales y subterráneas de la cuenca del río Oja (La Rioja, España): Vulnerabilidad del acuífero aluvial. Limnetica, 25(3): 753-762.

ARAUZO, M., J. A. DÍEZ, J. J. MARTÍNEZ-BASTIDA, M. VALLADOLID \& P. HERNÁIZ. 2007. Comparación de un método directo y un método indirecto para la estimación del balance hídrico en la zona no saturada. En: Estudios de la Zona no Saturada del Suelo, Vol. VIII, ZNS'07. J. V. Giráldez Cervera y F. J. Jiménez Hornero (eds.): 77-82. Universidad de Córdoba -CSIC- Junta de Andalucía.

ARAUZO, M., J. J. MARTÍNEZ-BASTIDA, M. VALLADOLID \& J. A. DÍEZ. 2010. Field evaluation of Gee Passive Capillary Lysimeters for monitoring drainage in nongravelly and gravelly alluvial soils: A useful tool to estimate nitrogen leaching from agriculture. Agricultural Water Management, 97: 465-474 (DOI: 10.1016/ j.agwat.2009.11.006)

ARMITAGE, P. D., MOSS, F., WRIGHT, J. F. \& M. T. FURSE. 1983. The preforman of a new biological water quality score system based on macroinvertebrates over a wide range of unpolluted running-water sites. Water Research, 17: 333-347.

BOLETÍN OFICIAL DEL ESTADO. 2008. Orden ARM/2658/2008 de 10 de septiembre, por la que se aprueba la instrucción de planificación hidrológica. $B O E \mathrm{n}^{\circ}$ 229: 38472-38582.

BOLETÍN OFICIAL DE LA RIOJA. 2001. Documento resumen del Plan Director de Saneamiento y Depuración de la Comunidad Autónoma de La Rioja 2000-2010. BOR $\mathrm{n}^{\circ}$ 124: 4416-4425.

CONFEDERACIÓN HIDROGRÁFICA DEL EBRO (CHE). 2004. Diseño de la Red de macroinvertebrados en la Cuenca del Ebro. Informe final. Diciembre 2004. $530 \mathrm{pp}$.

CONFEDERACIÓN HIDROGRÁFICA DEL EBRO (CHE). 2005 a. Metodología para el establecimiento del Estado Ecológico según la Directiva 
Marco del Agua. Protocolos de muestreo y análisis para invertebrados bentónicos. Comisaría del Agua, CHE, Ministerio de Medio Ambiente. Octubre 2005, $56 \mathrm{pp}$.

CONFEDERACIÓN HIDROGRÁFICA DEL EBRO (CHE). 2005 b. Explotación de la Red de macroinvertebrados en la Cuenca del Ebro. Informe final. Diciembre 2005. 484 pp.

CONFEDERACIÓN HIDROGRÁFICA DEL EBRO (CHE). 2006 a. Establecimiento de condiciones de referencia y redefinición de redes en la cuenca del Ebro, según la Directiva 2000/60/CE (Exp. $n^{\circ}$ 27/04-A).

CONFEDERACIÓN HIDROGRÁFICA DEL EBRO (CHE). 2006 b. Red de Control Biológico en ríos. Informe final Ríos, año 2006. $185 \mathrm{pp}$.

CONFEDERACIÓN HIDROGRÁFICA DEL EBRO (CHE). 2007. Red de Control Biológico en ríos. Informe final Ríos, año 2007. 472 pp.

CONFEDERACIÓN HIDROGRÁFICA DEL EBRO (CHE). 2008. Red de Control Biológico en ríos. Informe final Ríos, año 2008. 915 pp.

CONFEDERACIÓN HIDROGRÁFICA DEL EBRO, JUNTA DE CASTILLA Y LEÓN y GOBIERNO DE LA RIOJA. 2007. Borrador del Plan Hidrológico de la cuenca del río Oja-Tirón. 187 pp.

GALLARDO-MAYENCO, A., S. MACÍAS \& J. TOJA. 2004. Efectos de la descarga en la calidad del agua a lo largo de un río mediterráneo: el río Guadaira (Sevilla). Limnetica, 23(1-2): 65-78.

GASITH, A. \& V.H. RESH. 1999. Streams in Mediterranean climate regions: Abiotic influences and biotic responses to predictable seasonal events. Annu. Rev. Ecol. Syst., 30: 51-58.

GOBIERNO DE LA RIOJA (GLR). 2007. Desarrollo de los trabajos sobre la evaluación y mejora del estado de las masas de agua superficiales y subterráneas de la Comunidad Autónoma de La Rioja según la Directiva Marco del Agua (2000/60/CE). Cuenca Oja-Tirón. 72 pp.

GOBIERNO DE LA RIOJA (GLR). 2008. Plan Director de Saneamiento y Depuración 2007-2015 de la Comunidad Autónoma de La Rioja. 144 pp.

MARTÍNEZ-BASTIDA, J. J., M. ARAUZO \& M. VALLADOLID. 2006. Diagnóstico de la calidad ambiental del río Oja (La Rioja, España) mediante el análisis de la comunidad de macroinvertebrados bentónicos. Limnetica, 25: 733-743.

MARTÍNEZ-BASTIDA, J. J., M. ARAUZO y M. VALLADOLID. 2007. Caracterización hidroquími- ca de las aguas superficiales y subterráneas en la cuenca del Oja-Tirón. Procesos de contaminación. Limnetica, 26: 219-231.

MARTÍNEZ-BASTIDA, J. J., M. ARAUZO \& M. VALLADOLID. 2009 a. Effects of excess nitrogen fertilization on the quality of groundwater in the Oja Catchment (North of Spain): an approach through the application of the CROPSYST model. In: 16th Nitrogen Workshop: Connecting different scales of nitrogen use in agriculture. Turín, Italia. C. Grignani, M. Acutis, L. Zavattaro, L. Becheni, C. Bertola, M. Gallina \& D. Sacco (eds.): 543-544. Università Degli Studi Di Torino, Università Degli Studi Di Milano.

MARTÍNEZ-BASTIDA, J. J., M. ARAUZO \& M. VALLADOLID. 2009 b. Efecto de las propiedades físicas del suelo sobre su contenido en agua en áreas aluviales de uso agrícola de La Rioja Alta. En: Estudios de la Zona No Saturada del Suelo. Vol IX-ZNS'09. O. Silva \& J. Carrera (eds.): 296303. CIMNE. Barcelona.

MIMAN. 2005. Directiva 2000/60/CE. Caracterización de los tipos de ríos y lagos, v. 4.0 (Inédito). CEDEX y Ministerio de Medio Ambiente. Madrid, $251 \mathrm{pp}$.

OSCOZ, J., J. GOMÀ, L. ECTOR, J. CAMBRA, M. PARDOS \& C. DURÁN. 2007. Estudio comparativo del estado ecológico de los ríos de la cuenca del Ebro mediante macroinvertebrados y diatomeas. Limnetica, 26(1): 143-158

OSCOZ, J., C. DURÁN, M. PARDOS, J. GIL \& A. VIAMONTE. 2008. Evolución histórica de la calidad biológica del agua en la cuenca del Ebro (España)(1990-2005). Limnetica, 27: 119-130.

TACHET, H., P. RICHOUX, M. BOURNAUD \& P. USSEGLIO-POLATERA. 2003. Invertébrés d'eau douce. Systématique, biologie, écologie. CNRS Editions, Paris. 587 pp.

VALLADOLID, M., J. J. MARTÍNEZ-BASTIDA, M. ARAUZO \& C. GUTIÉRREZ. 2006. Abundancia y biodiversidad de los macroinvertebrados del río Oja (La Rioja, España). Limnetica, 25: 745-751.

VALLADOLID, M., J. J. MARTÍNEZ-BASTIDA \& M. ARAUZO. 2007. Los Hydropsychidae (Insecta: Trichoptera) del río Oja (La Rioja, Spain). Limnetica, 26: 199-208.

VALLADOLID, M., J. J. MARTÍNEZ-BASTIDA \& M. ARAUZO. 2010. The Trichoptera fauna of the Oja River (La Rioja, Spain). Zoosymposia (en prensa). 